\title{
Association of Proteinuria and Hematuria with Acute Kidney Injury and Mortality in Hospitalized Patients with COVID-19
}

\author{
Imran Chaudhri ${ }^{a}$ Richard Moffitt $^{b}$ Erin Taub ${ }^{a}$ Raji R. Annadic \\ Minh Hoai ${ }^{c}$ Olena Bolotova ${ }^{a}$ Jeanwoo Yoo ${ }^{a}$ Simrat Dhaliwal ${ }^{a}$ \\ Haseena Sahib ${ }^{a}$ Farah Daccueil $^{a}$ Janos Hajagos $^{b}$ Mary Saltz $^{b}$ \\ Joel Saltz ${ }^{b}$ Sandeep K. Mallipattu ${ }^{a, d}$ Farrukh M. Koraishy ${ }^{a, d}$ \\ a Division of Nephrology and Hypertension, Department of Medicine, Stony Brook University, \\ Stony Brook, NY, USA; ${ }^{b}$ Department of Biomedical Informatics, Stony Brook University, \\ Stony Brook, NY, USA; ' Department of Computer Science, Stony Brook University, \\ Stony Brook, NY, USA; dRenal Section, Northport VA Medical Center, Northport, NY, USA
}

\section{Keywords}

COVID-19 · Hematuria $\cdot$ Proteinuria · Acute kidney injury

\begin{abstract}
Introduction: Acute kidney injury (AKI) is strongly associated with poor outcomes in hospitalized patients with coronavirus disease 2019 (COVID-19), but data on the association of proteinuria and hematuria are limited to non-US populations. In addition, admission and in-hospital measures for kidney abnormalities have not been studied separately. Methods: This retrospective cohort study aimed to analyze these associations in 321 patients sequentially admitted between March 7, 2020 and April 1, 2020 at Stony Brook University Medical Center, New York. We investigated the association of proteinuria, hematuria, and AKI with outcomes of inflammation, intensive care unit (ICU) admission, invasive mechanical ventilation (IMV), and in-hospital death. We used ANOVA, $t$ test, $\chi^{2}$ test, and Fisher's exact test for bivariate analyses and logistic regression for multivariable analysis. Results: Three hundred patients met the inclusion criteria for the study cohort. Multivariable analysis demonstrated that admission proteinuria was significantly associated with risk of in-hospital AKI (OR 4.71, 95\% Cl 1.28-17.38), while admission hematuria was associated with ICU admission (OR 4.56, 95\% Cl 1.12-18.64), IMV (OR 8.79, 95\% Cl 2.08-37.00), and death (OR 18.03, 95\% Cl 2.84-114.57). During hospitalization, de novo proteinuria was significantly associated with increased risk of death (OR $8.94,95 \%$ $\mathrm{Cl} 1.19-114.4, p=0.04$ ). In-hospital AKI increased (OR 27.14, 95\% Cl 4.44-240.17) while recovery from in-hospital AKI decreased the risk of death (OR 0.001, 95\% CI 0.001-0.06). Conclusion: Proteinuria and hematuria both at the time of admission and during hospitalization are associated with adverse clinical outcomes in hospitalized patients with COVID-19.
\end{abstract}

\section{Karger $\stackrel{2}{\circ}$}




\section{Introduction}

Coronavirus disease 2019 (COVID-19), caused by the novel severe acute respiratory syndrome coronavirus 2 (SARS-CoV-2), has rapidly evolved into a global pandemic associated with devastating morbidity and mortality in hospitalized patients [1]. COVID-19 is not only a lung disease [2] but also has a deleterious impact on multiple organ systems, including cardiovascular [3], nervous [4], coagulation [5], and skin [6], in the setting of severe inflammation and cytokine release [7]. Acute kidney injury (AKI) has now become a hallmark of COVID-19 and a major risk factor associated with mortality in hospitalized patients [8-10].

Some initial studies from China showed a variable prevalence of AKI in patients with COVID-19 $[11,12]$. More recent data from the United States show a much higher prevalence [13]. Hirsch et al. [9] conducted a large study in metropolitan New York and found that almost $40 \%$ of patients developed AKI, which was strongly associated with mechanical ventilation and mortality, especially in patients admitted to the intensive care unit (ICU).

Proteinuria and hematuria are reported to have an even higher prevalence in COVID-19 than AKI [14]. Retrospective studies in China have reported that proteinuria and hematuria are associated with COVID-19 [15] and with higher mortality [8, 14]. To our knowledge, proteinuria and hematuria and their associated outcomes have not been reported in hospitalized patients with COVID-19 in the United States. While previous studies have analyzed kidney disease in hospitalized patients with COVID-19, they did not differentiate the outcomes associated with kidney abnormalities at presentation (admission) versus those that occur during hospitalization.

We hypothesized that proteinuria and hematuria present at the time of admission or occurring de novo during hospitalization will be independently associated with adverse outcomes in patents with COVID-19. We report the clinical characteristics and laboratory data of hospitalized COVID-19 patients at Stony Brook University Medical Center in Long Island, New York and analyze the risk of proteinuria, hematuria, and AKI and their impact on clinical outcomes.

\section{Materials and Methods}

\section{Study Design and Participants}

We conducted a retrospective cohort study on patients hospitalized with COVID-19 at Stony Brook University Medical Center from March 7, 2020 to April 1, 2020 during the initial COVID-19 outbreak. COVID-19 was confirmed by at least one positive result for SARS-CoV-2 on PCR testing of nasopharyngeal samples. Patients were followed up until a final disposition of discharge alive from the hospital or in-hospital death. We excluded patients $<18$ years of age, those with end-stage kidney disease, with solid organ transplants, and with a history of immunosuppressant medication use.

\section{Data Collection and Definition of Variables}

Using the electronic health record (EHR), data on race, ethnicity, age, sex, and body mass index were collected. Hemoglobin A1c $>6.5 \%$ or treatment with insulin or oral hypoglycemics prior to hospitalization was used to determine diabetes. Coronary artery disease was identified from previous cardiac catheterization reports. "Vascular disease" was defined as atherosclerotic disease other than coronary artery disease, including cerebrovascular disease, carotid artery stenosis, or peripheral artery disease. Heart failure was determined by presence of systolic dysfunction, diastolic dysfunction, or both on transthoracic echocardiogram. Chronic kidney disease (CKD) was diagnosed through documentation in the EHR and/or

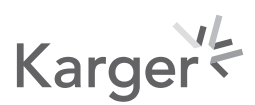


International Classification of Diseases (ICD)-9/10 codes. Chronic obstructive pulmonary disease was defined using the EHR, ICD-9/10 codes, and prior pulmonary function tests. Pulmonary diseases other than chronic obstructive pulmonary disease, including asthma, obstructive sleep apnea, pulmonary hypertension, and lung malignancy, were included in the term "other lung diseases" and were identified using EHR documentation or ICD-9/10 codes. The EHR was used to document a history of angiotensin-converting enzyme inhibitors and angiotensin receptor blocker use. The triage vital signs on presentation to the emergency department were used to identify the baseline systolic and diastolic blood pressure and the mean arterial pressure. Outpatient use of antihypertensive medications or sustained systolic blood pressure $>140 \mathrm{~mm} \mathrm{Hg}$ and diastolic blood pressure $>80 \mathrm{~mm} \mathrm{Hg}$ on admission were used to determine hypertension. If intravenous fluids were administered within the first $12 \mathrm{~h}$ of presentation to the hospital, we also collected those data.

\section{Proteinuria and Hematuria}

Proteinuria was defined by presence of $\geq 1+$ protein on urinalysis, hematuria was defined as the presence of $>4$ red blood cells per high-power field on urinalysis, and urinary tract infection was defined as presence of a positive urine culture. Patients were categorized as having proteinuria or hematuria "on admission" if they had these findings within the first $48 \mathrm{~h}$ of presentation to the emergency department. If these findings appeared on later urinalyses, they were defined as "in-hospital" values. Admission proteinuria was analyzed in a cohort that excluded patients with in-hospital proteinuria, while in-hospital proteinuria was analyzed in a cohort that excluded patients with admission proteinuria. Cohorts for admission and in-hospital hematuria were analyzed similarly. A smaller subset of these cohorts included patients who had both proteinuria and hematuria, termed "combined proteinuria/hematuria," who were compared to those who never developed proteinuria or hematuria.

Data on indwelling Foley catheter placement and anticoagulation use were also documented as these factors could influence in-hospital proteinuria and hematuria measurements. Review of documentation and the EHR was done for each patient to see whether they had an indwelling Foley catheter placed during the hospitalization prior to recording of in-hospital hematuria or proteinuria. The EHR was also reviewed to determine whether patients received any amount of therapeutic anticoagulation during the hospitalization. Deep vein thrombosis chemical prophylactic dosing of heparin or enoxaparin was not considered therapeutic anticoagulation.

Only 22 patients out of the 300 (7.33\%) had urinalysis data recorded in the EHR in a 365-day period prior to admission. As this number was too small, we did not include these data in our analysis.

\section{Acute Kidney Injury}

In patients with a documented baseline serum creatinine (SCr) within 3 months prior to admission, "AKI on admission" was defined as a rise in $\mathrm{SCr}$ of $0.3 \mathrm{mg} / \mathrm{dL}$ or an increase 1.5 times the baseline based on the Kidney Disease: Improving Global Outcomes (KDIGO) definition [16]. For patients without a 3-month baseline SCr, "AKI on admission" was retroactively defined as a $\mathrm{SCr}>1.2 \mathrm{mg} / \mathrm{dL}$ (upper limit of normal in Stony Brook University Medical Center Lab) within the first $48 \mathrm{~h}$ of presentation to the emergency department with subsequent improvement by $50 \%$ during the hospitalization [14]. "In-hospital AKI" was diagnosed based on KDIGO in those without admission AKI. Renal replacement therapy was defined as the need for either hemodialysis, continuous kidney replacement therapy, or both. Admission AKI was analyzed in a cohort that excluded patients with in-hospital AKI, while in-hospital AKI was analyzed in a cohort that excluded patients with admission AKI. Patients who met the criteria for both admission and in-hospital AKI were not included in either of the AKI

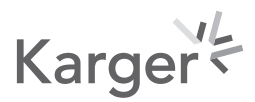


analyses so that differences between admission and in-hospital AKI could be more clearly delineated.

AKI classes for in-hospital AKI were assigned to all patients based on the Acute Kidney Injury Network (AKIN) criteria [17]. Class 1 was assigned for an acute increase in SCr by 50\%, class 2 for an increase by $100 \%$, and class 3 for an increase of $200 \%$ or if the patient required renal replacement therapy during the hospitalization [17]. "Recovery from AKI" was defined as improvement to the patient's baseline or normal SCr [18].

\section{Laboratory Data}

"Admission" laboratory data were defined as data collected in the first $48 \mathrm{~h}$ of presentation and included SCr, blood urea nitrogen (BUN), potassium, N-terminal prohormone of brain natriuretic peptide (NT-proBNP), estimated glomerular filtration rate (eGFR), ferritin, lactate dehydrogenase (LDH), erythrocyte sedimentation rate (ESR), D-dimer, and C-reactive protein (CRP). eGFR was determined using the CKD-Epi equation [19]. Two composite scores for inflammation were calculated, named "peak inflammation score" and "fold change inflammation score," based on the available laboratory data using methods based on previous reported studies [20] and explained in our recent study [21]. "Peak" laboratory values for SCr, ferritin, LDH, ESR, D-dimer, and CRP were also collected during the hospital course.

\section{Outcomes}

The data on outcomes of ICU admission, invasive mechanical ventilation (IMV), and in-hospital death were obtained from EHR documentation.

\section{Statistical Analysis}

"Admission" values for AKI, proteinuria, and hematuria were all analyzed against outcomes of ICU admission, IMV, and death, while "in-hospital" kidney abnormalities were analyzed against the outcome of death alone. Statistical analysis was conducted in R 3.6.0, including the core package stats [22]. Continuous variables were reported as mean (standard deviation) or median (interquartile range) depending on normality. These variables were then compared by either an independent $t$ test or ANOVA for parametric variables and Wilcoxon rank sum or Kruskal-Wallis test for nonparametric variables. Categorical variables were compared by either a $\chi^{2}$ test or Fisher's exact test as appropriate when data were sparse. Logistic regression was used for multivariable analyses with dichotomous outcomes after removing highly correlated features identified from bivariate study. These models represented a combination of inferential and descriptive analyses. A $p$ value $<0.05$ was considered statistically significant.

\section{Results}

\section{Baseline Characteristics}

Of the 321 RT-PCR-confirmed SARS-CoV-2 sequentially hospitalized patients aged $\geq 18$ years, 300 patients who met the inclusion criteria were included in the study (online suppl. Fig. 1; for all online suppl. material, see www.karger.com/doi/10.1159/000511946), of whom $28 \%$ had proteinuria, $19 \%$ had hematuria, $9 \%$ had combined proteinuria/hematuria, and $27 \%$ had AKI. The mean age was $59.3 \pm 17.7$ years, with 133 patients $(44.3 \%)$ with a history of hypertension and 72 patients $(24.0 \%)$ with diabetes (online suppl. Table 1 ). In the study cohort, $38.7 \%$ had more than one comorbidity. Most patients were Caucasian (81.9\%) and a significant proportion were Hispanic (33.7\%).

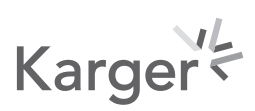


Kidney and

Blood Pressure

Research
Kidney Blood Press Res 2020;45:1018-1032

1022

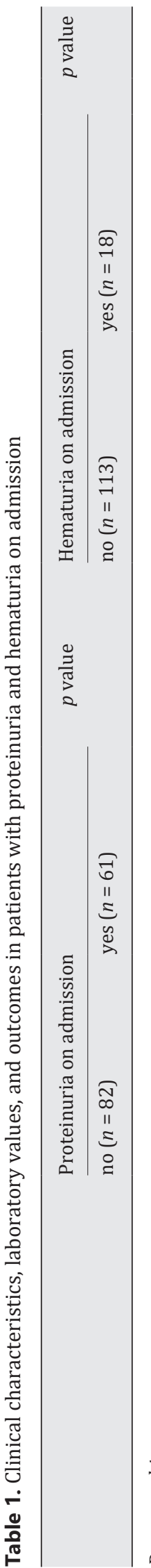

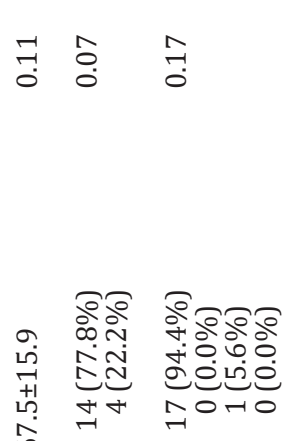

\section{อ.}

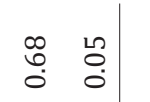

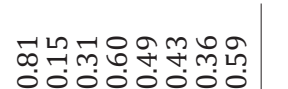

웅요

00000000

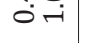

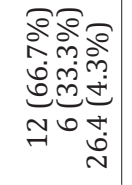

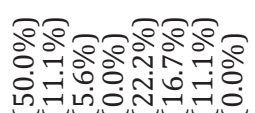

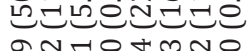

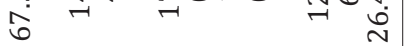

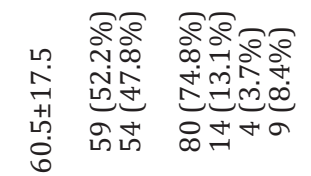

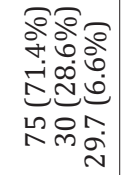

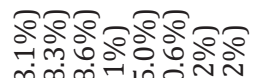

min

Вกतั

www.karger.com/kbr

Chaudhri et al.: Proteinuria and Hematuria in COVID-19

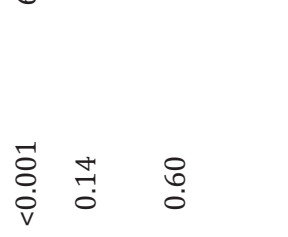

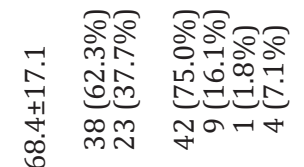

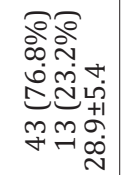

คำำำ

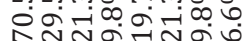

mom $m$ m

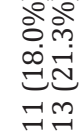

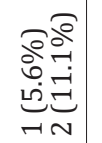

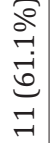

mิำ ำ

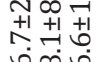

mino

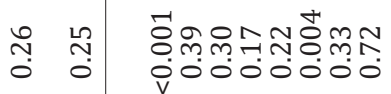

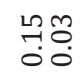

\section{จㅇำ \\ เి่}

듬ㄷㅁ

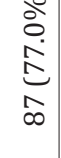

กิ่งอ

影

ิㅜㄱำ

iิ่

눙서워ำ

+1

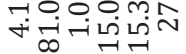

ํㅗㅇ

(a)

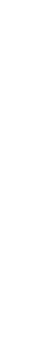

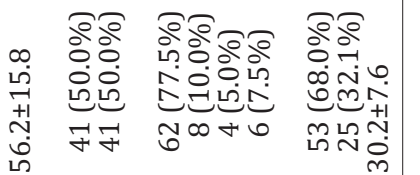

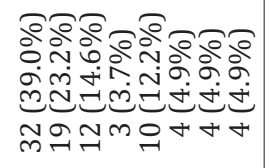

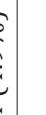

\section{จㅇำ \\ ติ \\ $\infty N$}

\section{ำ
0
0
0
0
0}

กุํา

$\stackrel{+1}{+1}+\frac{1}{+1}$

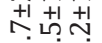

กี่ฐ்

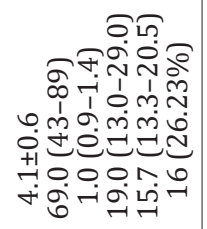

\section{อำ}

नำกำ

1ำ 응ำ

- $0.0 \mathrm{~m}$

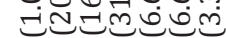

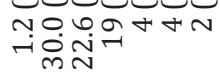

लिंतू

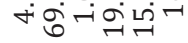

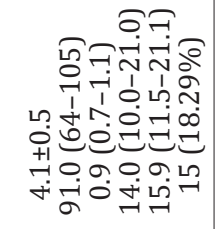

จำ

त.

సं

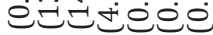

즈융
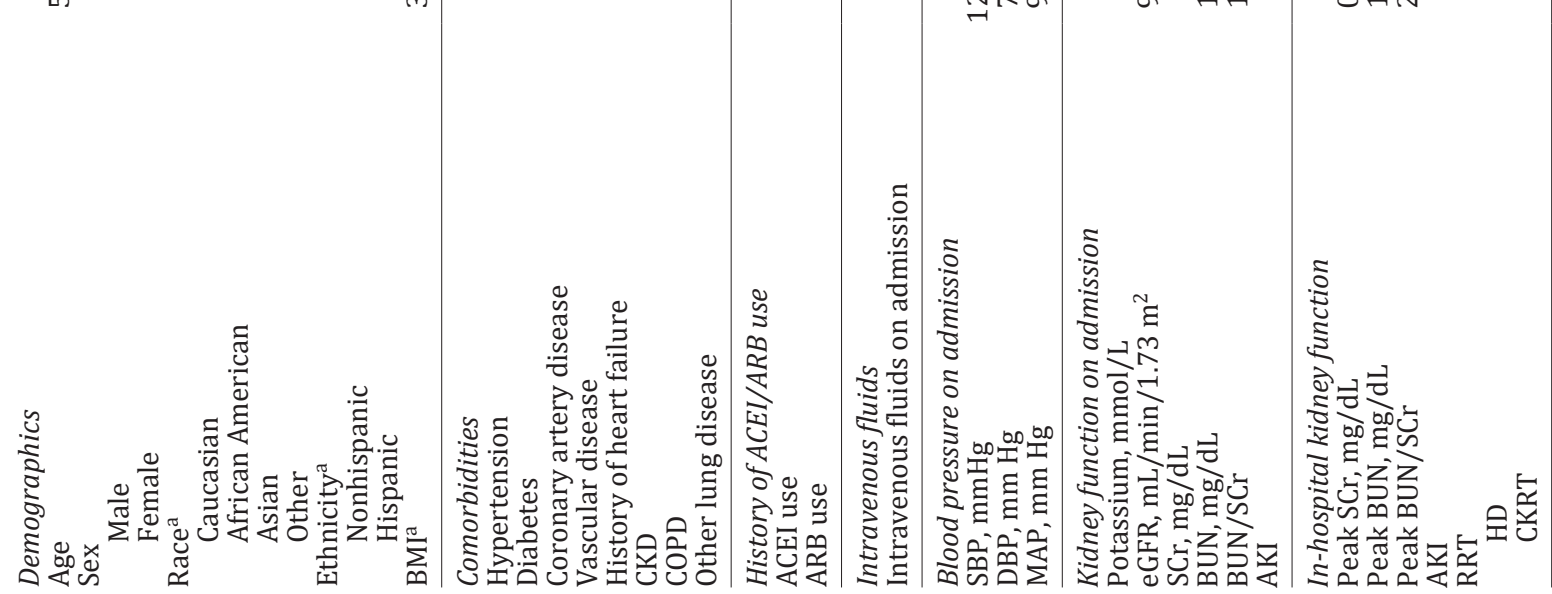
Kidney and

Blood Pressure

Research
Kidney Blood Press Res 2020;45:1018-1032

Chaudhri et al.: Proteinuria and Hematuria in COVID-19

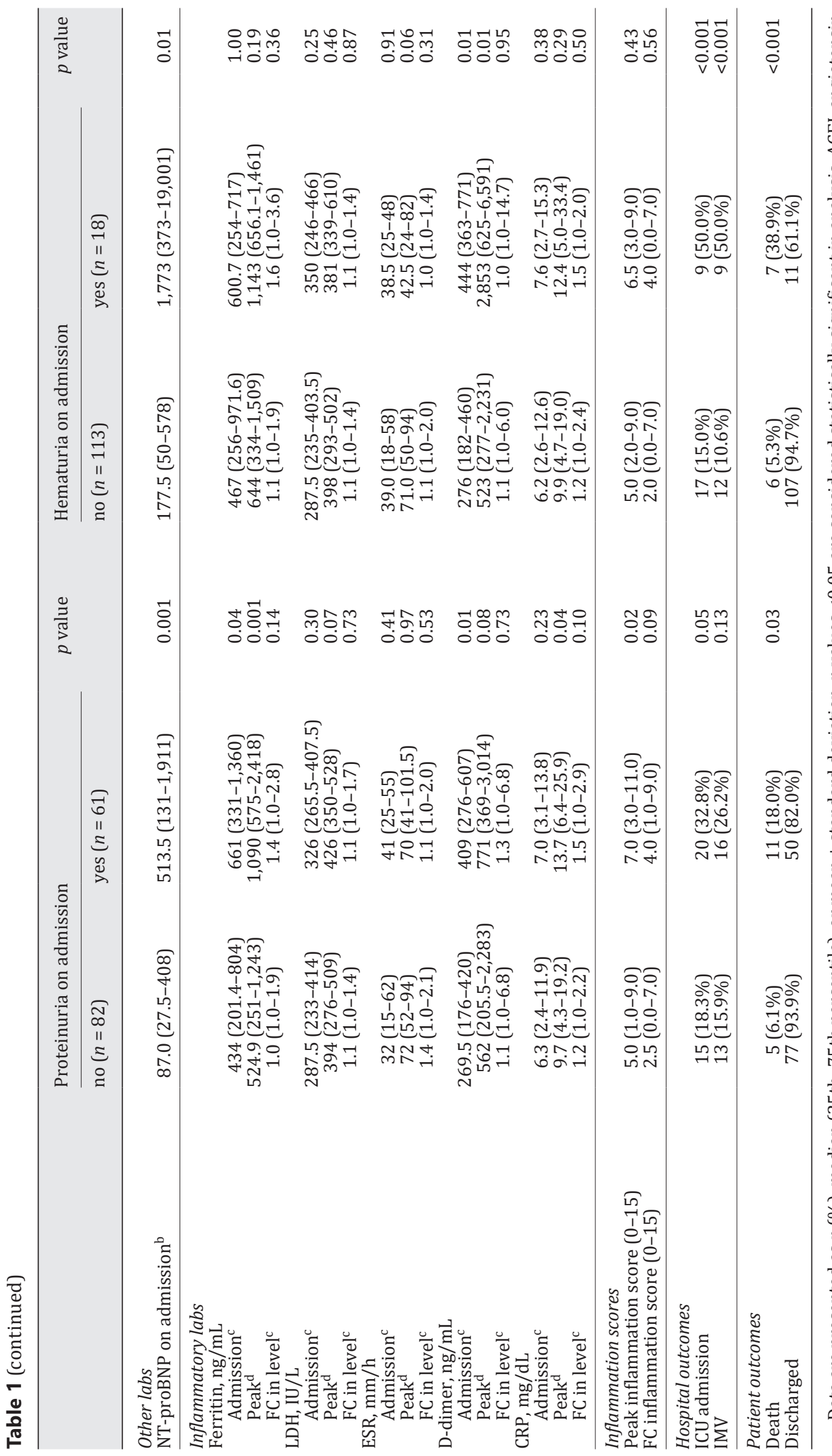

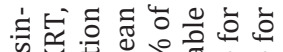

记

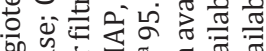

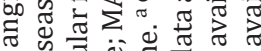

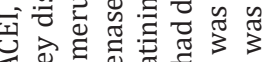

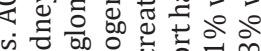
ज记

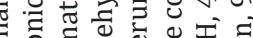
ב 过 热壬 언

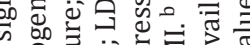

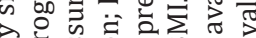
तٓ क 屯 응 त 응

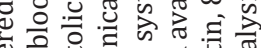

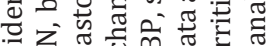

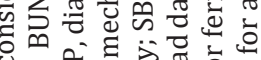

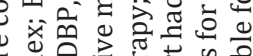

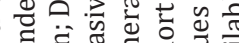

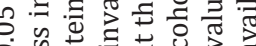
品

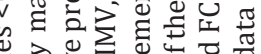

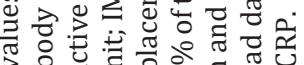

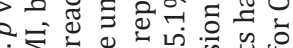

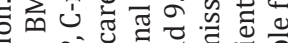

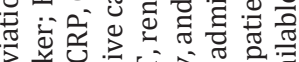

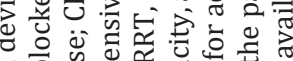
긍 के व

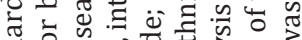

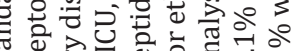

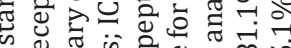

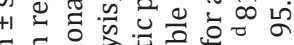

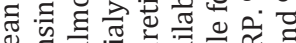
चี 응

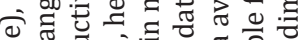

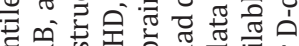
व 定.

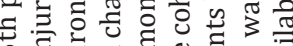

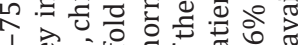
1 बें

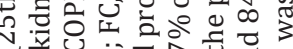
च 过灾 च च च हैं

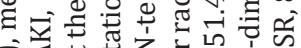

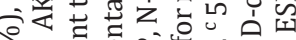

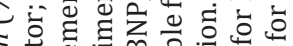

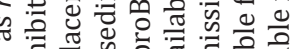
更 하워 है

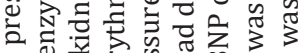

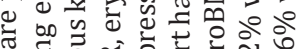

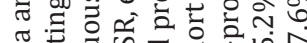

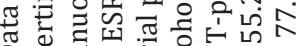

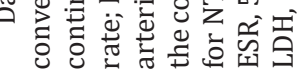




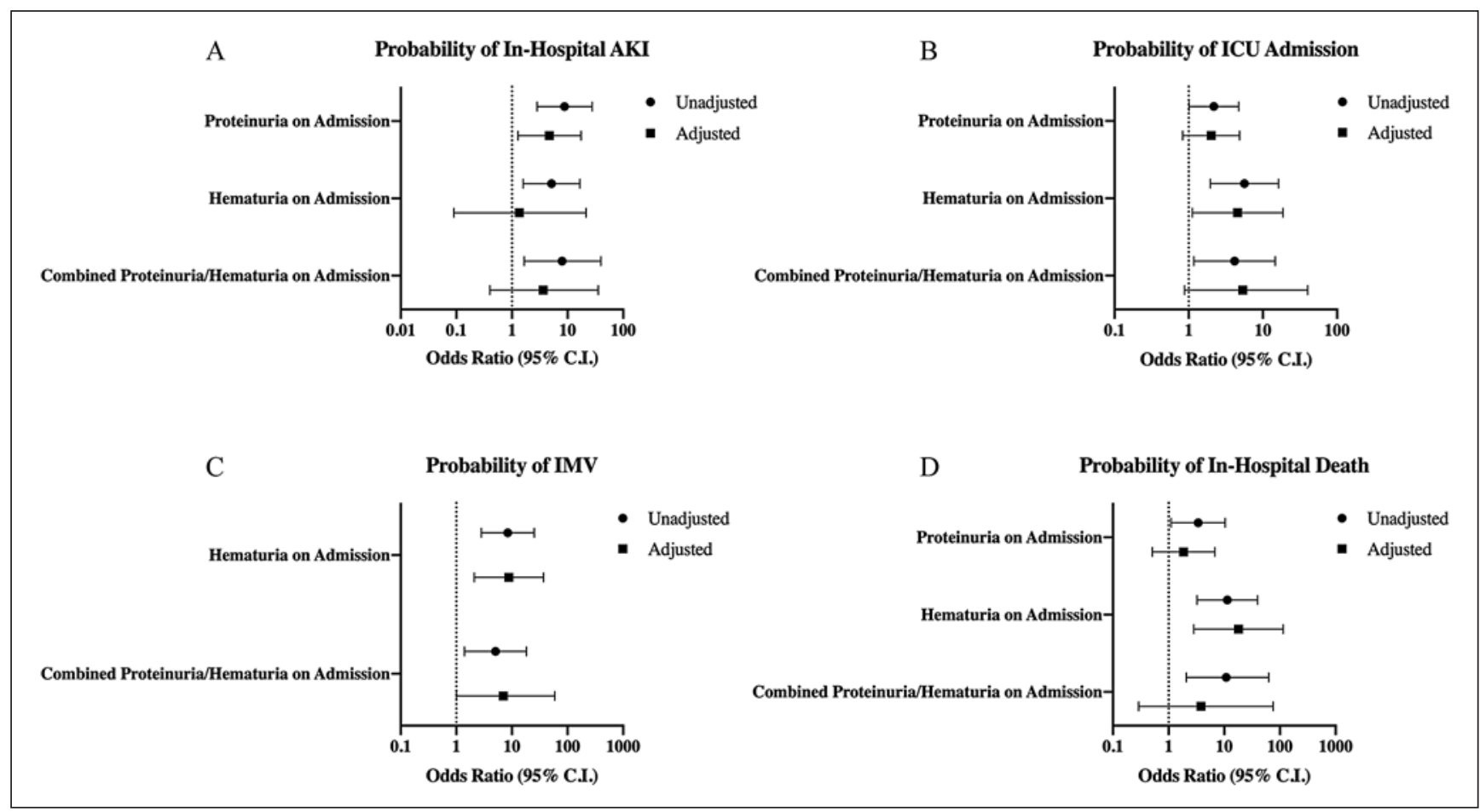

Fig. 1. Forest plots for unadjusted and adjusted ORs and 95\% CIs for admission values for proteinuria and hematuria against outcomes of in-hospital AKI (A), ICU admission (B), IMV (C), and in-hospital death (D). All "proteinuria on admission" analyses were adjusted for covariates of age, hypertension history, CKD history, history of angiotensin receptor blocker use, systolic blood pressure on admission, eGFR on admission, and SCr on admission. All "hematuria on admission" analyses were adjusted for covariates of diastolic blood pressure on admission, BUN/SCr on admission, and peak BUN. Lastly, all "combined proteinuria/hematuria on admission" analyses were adjusted for covariates of age, systolic blood pressure on admission, peak BUN, peak ferritin, and peak D-dimer level. Logistic regression was used for multivariable analyses with dichotomous outcomes after removing highly correlated features identified from bivariate study to obtain adjusted ORs and 95\% CIs. All graphs show independent variables grouped on the $y$ axis with ORs and 95\% CI graphed on the $x$ axis. Error bars represent 95\% CI for each variable. AKI, acute kidney injury; BUN, blood urea nitrogen; CKD, chronic kidney disease; eGFR, estimated glomerular filtration rate; ICU, intensive care unit; IMV, invasive mechanical ventilation; $\mathrm{SCr}$, serum creatinine.

Proteinuria and hematuria data were not analyzed for 131 patients (43.7\%) as 103 patients (34.3\%) did not have a urinalysis done during their hospitalization and 28 patients (9.3\%) had a urinary tract infection. At admission only, 61 (20.3\%) patients had proteinuria, $18(6.0 \%)$ had hematuria, and $13(4.3 \%)$ had combined proteinuria/hematuria. During hospitalization only, 23 (7.7\%), 38 (12.7\%), and 15 (5.0\%) patients developed proteinuria, hematuria, and combined proteinuria/hematuria, respectively. Thirty-five patients $(11.7 \%)$ had AKI at admission, $28(9.3 \%)$ during hospitalization, and $19(6.3 \%)$ met the criteria for both AKI on admission and in-hospital AKI.

Of the 300 patients, admission laboratory data were not available for ferritin (143, 47.7\%), LDH (42, 14\%), ESR (160, 53.3\%), D-dimer (122, 40.7\%), and CRP (41, 13.7\%).

\section{Proteinuria and Hematuria on Admission}

Table 1 shows the demographics, laboratory data, and clinical outcomes of patients with admission proteinuria and hematuria, respectively. 
Patients with proteinuria at admission were older and had a higher prevalence of hypertension, CKD, and angiotensin receptor blocker use compared to those without. Admission ferritin, D-dimer, NT-proBNP, and peak values for BUN, ferritin, CRP, and inflammation score were associated with proteinuria (Table 1). Those with admission proteinuria had a higher incidence of in-hospital AKI, ICU admission, and death compared to those without proteinuria (Table 1). After controlling for multiple covariates, admission proteinuria remained significantly associated with in-hospital AKI (OR 4.71, 95\% CI 1.28-17.38, $p=0.02$ ) (Fig. 1).

Admission hematuria was associated with increased baseline D-dimer, NT-proBNP, and peak BUN. Those with hematuria at admission had a higher incidence of in-hospital AKI, ICU admission, IMV, and death compared to those without hematuria (Table 1). On multivariable analysis, admission hematuria remained significantly associated with ICU admission (OR 4.56, 95\% CI 1.12-18.64, $p=0.03$ ), IMV (OR 8.79, 95\% CI 2.08-37.00, $p=0.003$ ), and death (OR 18.03, 95\% CI 2.84-114.57, $p=0.002$ ) (Fig. 1).

Combined proteinuria/hematuria was associated with an increased risk of ICU admission, IMV, and death (online suppl. Table 2). These relationships did not show statistical significance on multivariable analysis, although there was a trend toward significance for ICU admission (OR 5.35, 95\% CI 0.88-40.24, $p=0.08$ ) and IMV (OR 6.99, 95\% CI 1.08-59.12, $p=0.05$ ) (Fig. 1).

\section{In-Hospital Proteinuria and Hematuria}

Table 2 shows differences in demographics, laboratory data, and clinical outcomes for patients with in-hospital proteinuria and hematuria, respectively.

Patients with in-hospital proteinuria had higher LDH, while those with in-hospital hematuria had higher LDH, D-dimer, and CRP on admission. Patients with proteinuria, hematuria, and combined proteinuria/hematuria during hospitalization were more likely to be male and had an independent association with in-hospital AKI, indwelling Foley catheter use, use of anticoagulation, increased inflammatory scores, ICU admission, IMV, and death (Table 2; online suppl. Table 3).

On multivariable analysis, in-hospital proteinuria remained significantly associated with mortality (OR 8.94, 95\% CI 1.19-114.4, $p=0.04$ ), while hematuria and combined proteinuria/ hematuria did not (Fig. 2).

\section{AKI on Admission}

Online supplementary Table 4 shows differences in demographics, laboratory data, and clinical outcomes for patients with admission AKI. Thirty-five patients (13.8\%) met the criteria for AKI on admission, which was not significantly associated with outcomes of interest, and 33 (94\%) had complete recovery.

Multivariable analysis for predictors of admission AKI showed that Hispanic ethnicity had a lower risk (OR $0.20,95 \%$ CI 0.06-0.75), while history of hypertension was associated with increased risk of AKI on admission (OR 3.32, 95\% CI 1.02-10.8) (online suppl. Table 5).

\section{In-Hospital AKI}

Online supplementary Tables 6, 8, and 9 show differences in demographics, laboratory data, and clinical outcomes for patients with in-hospital AKI, AKIN classes, and recovery, respectively. Twenty-eight patients (11.4\%) met the criteria for in-hospital AKI, and of those $28.6 \%, 42.9 \%$, and $28.6 \%$ had AKIN classes 1, 2, and 3, respectively.

On multivariable analysis, history of hypertension (OR 6.53, 95\% CI 1.27-42.74) and admission CRP (OR 1.13,95\% CI 1.05-1.23) (online suppl. Table 7) were significant predictors, and in-hospital AKI was significantly associated with in-hospital death (OR 27.14, 95\% CI 4.44-240.17, $p=0.009$ ) (online suppl. Fig. 2).

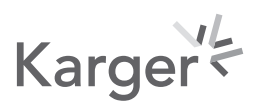


Kidney and

Blood Pressure

Research
Kidney Blood Press Res 2020;45:1018-1032

1026

\begin{tabular}{l|l}
\hline DOI: $10.1159 / 000511946$ & $\begin{array}{l}\odot 2 \text { 2020 The Author(s). Published by S. Karger AG, Basel } \\
\text { www.karger.com/kbr }\end{array}$ \\
\hline
\end{tabular}

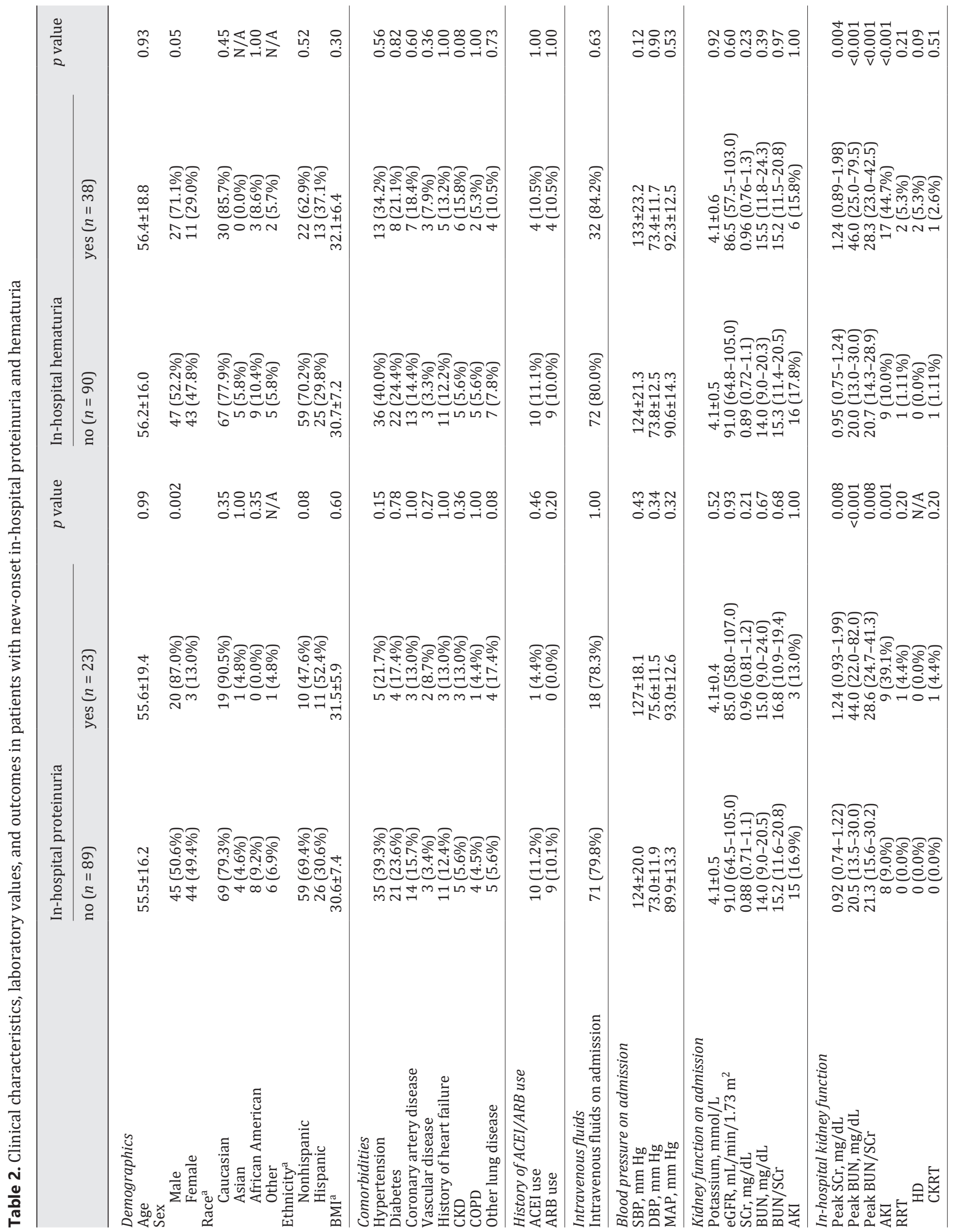


Kidney and

Blood Pressure

Research
Kidney Blood Press Res 2020;45:1018-1032

1027

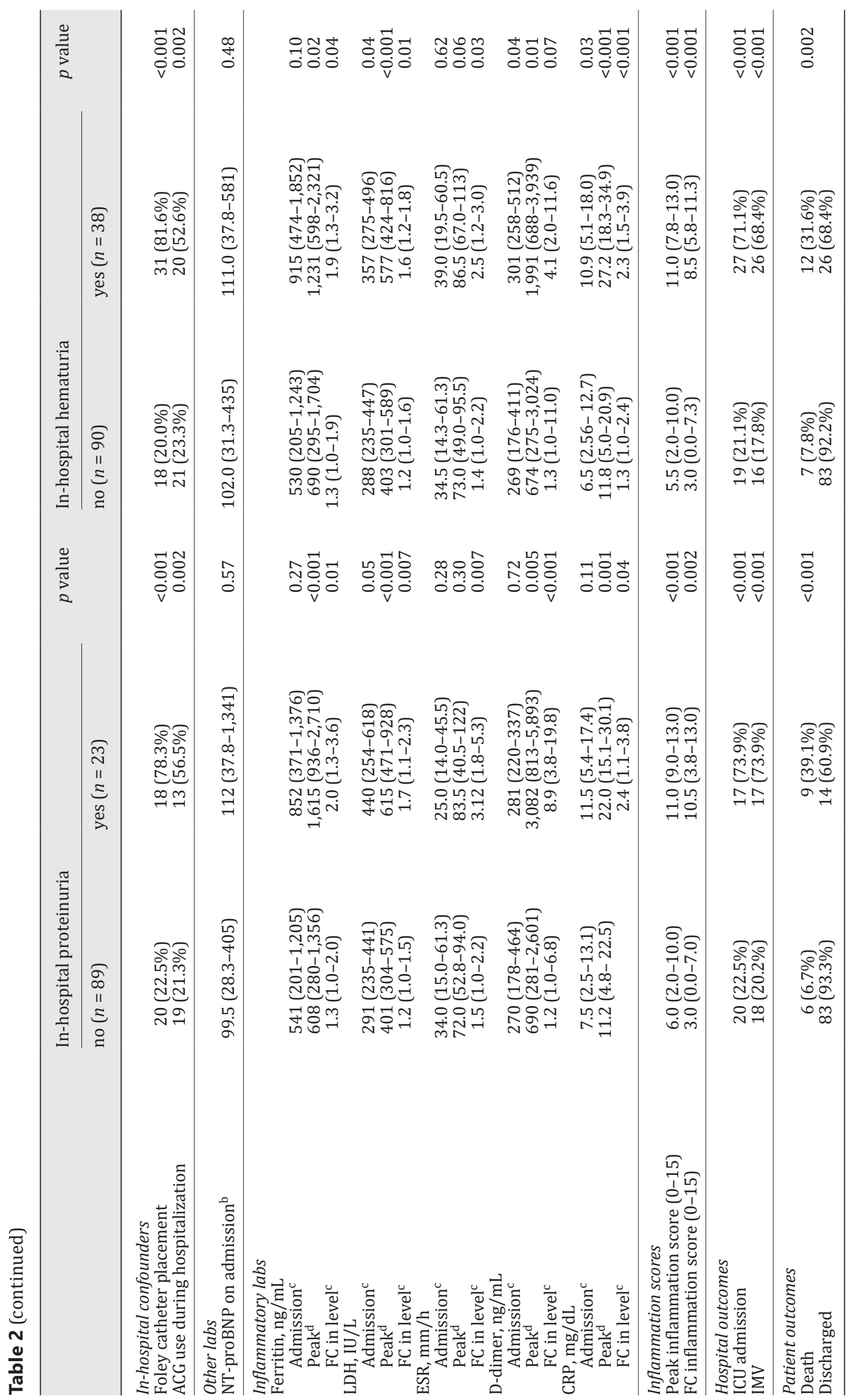

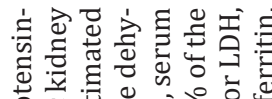

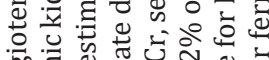

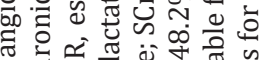
곤 यิ

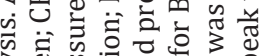

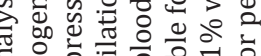
을

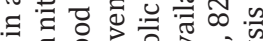
焉응 중 ฮึ

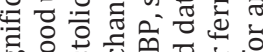

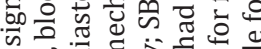

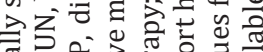

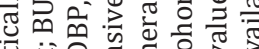

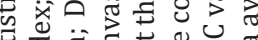

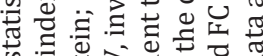

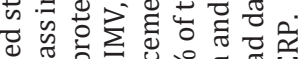
记

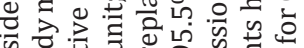
워의

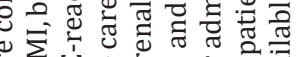

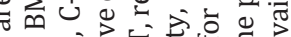

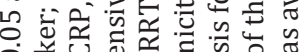
v ४ㅇํㅇ

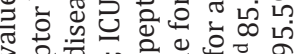

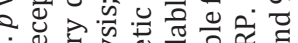
$\dot{1}$ 흔 㻤 政

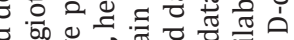

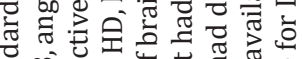

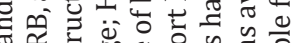

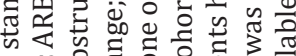
+1 응 준 च.

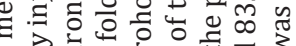

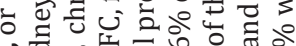

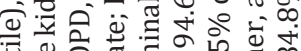

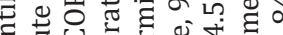

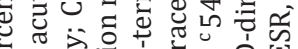

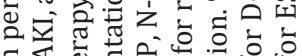

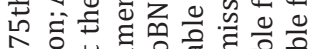
过

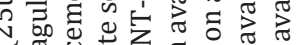

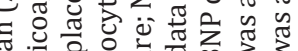
记 당

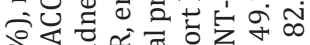

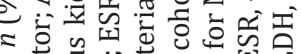

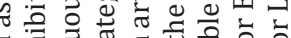
跣 可 हो

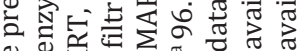
닌

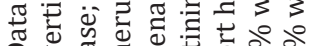

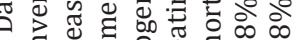

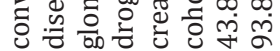




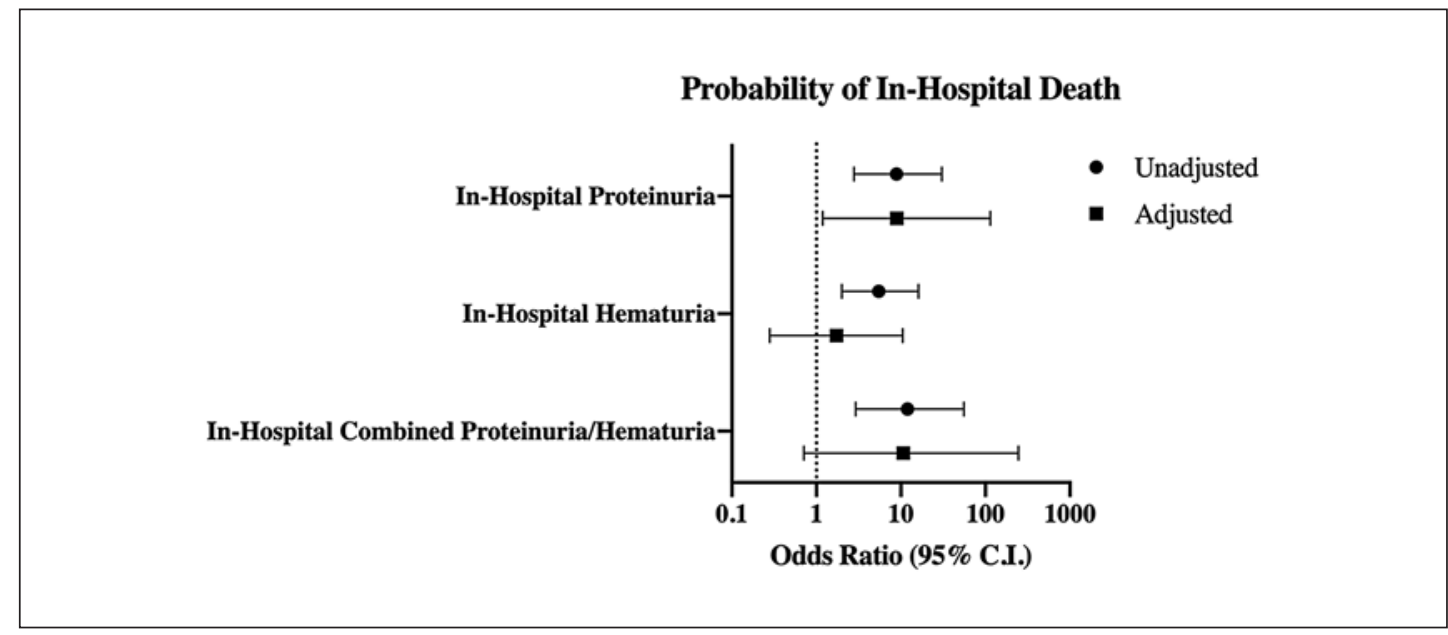

Fig. 2. Forest plot for unadjusted and adjusted ORs and 95\% CIs for in-hospital values for proteinuria and hematuria against outcome of in-hospital death. Analysis for "in-hospital proteinuria" was adjusted for covariates of sex, peak SCr, peak BUN, peak BUN/SCr, in-hospital AKI, indwelling Foley catheter use, use of anticoagulation, peak inflammation score, fold change inflammation score, and IMV. Analysis for "in-hospital hematuria" was adjusted for covariates of peak BUN/SCr, CRP on admission, in-hospital AKI, indwelling Foley catheter use, use of anticoagulation, peak inflammation score, fold change inflammation score, and IMV. Lastly, analysis for in-hospital "combined proteinuria/hematuria" was adjusted for covariates of sex, peak BUN/SCr, in-hospital AKI, indwelling Foley catheter use, use of anticoagulation, peak inflammation score, fold change inflammation score, and IMV. Logistic regression was used for multivariable analyses with dichotomous outcomes after removing highly correlated features identified from bivariate study to obtain adjusted ORs and 95\% CIs. Independent variables are grouped on the $y$ axis with ORs and 95\% CI graphed on the $x$ axis. Error bars represent 95\% CI for each variable. AKI, acute kidney injury; BUN, blood urea nitrogen; $\mathrm{CRP}, \mathrm{C}$-reactive protein; IMV, invasive mechanical ventilation; $\mathrm{SCr}$, serum creatinine.

Among the patients with in-hospital AKI, 17 (61\%) experienced complete recovery. On multivariable analysis, recovery was associated with decreased risk of in-hospital death (OR $0.001,95 \%$ CI $0.001-0.06, p=0.01$ ) (online suppl. Fig. 2).

\section{Discussion/Conclusions}

To our knowledge, this is the first report of the association of hematuria and proteinuria with adverse outcomes including death in a US population of hospitalized patients with COVID-19. We made the following novel observations in this study: (1) Admission proteinuria was associated with AKI during hospitalization. (2) Admission hematuria was associated with ICU admission, IMV, and death. (3) Proteinuria during hospitalization was significantly associated with increased risk of death. Consistent with previous studies, we also reported that in-hospital AKI was associated with a history of hypertension and with increased risk of death. Recovery from AKI reduced the risk of death.

As reported in non-US cohorts $[8,14]$, we observed a high prevalence of hematuria and proteinuria in patients hospitalized with COVID-19. We noted that even after adjustment of significant baseline differences, proteinuria and hematuria at admission significantly influenced in-hospital outcomes. Those with admission proteinuria had a 4.7 times higher risk of in-hospital AKI, while those with admission hematuria had a 4.6 times higher risk of ICU admission, an 8.8 times higher risk of requiring IMV, and an 18 times higher risk of death. Our 
data support data from other non-US cohorts $[8,14]$, emphasizing the importance of admission urinalysis in COVID-19. Pei et al. [14] analyzed 333 Chinese patients hospitalized with COVID-19 and observed that those with renal involvement (hematuria, proteinuria, or AKI) had higher hospital mortality than those without (11.2 vs. 1.2\%). In another study of 701 Chinese patients hospitalized with COVID-19, Cheng et al. [8] reported that proteinuria and hematuria were associated with an increased risk of in-hospital death after adjusting for age, sex, disease severity, comorbidity, and leukocyte count. These studies did not report the association of other outcomes such as inflammation, ICU admission, or IMV as our study.

To our knowledge, there are no reports of the outcomes associated with de novo proteinuria and hematuria that occur during hospitalization for COVID-19. Hirsch et al. [9] reported that both proteinuria and hematuria were common $24 \mathrm{~h}$ before and up to $48 \mathrm{~h}$ after the development of AKI, but no independent association with outcomes was reported. For in-hospital proteinuria and hematuria, we only tested the outcome of hospital death since other outcomes sometimes preceded the onset of proteinuria or hematuria. In-hospital proteinuria was significantly associated with death, with a risk of 8.9 times compared to those without proteinuria. This important finding again highlights the significance of urinalysis in patients with COVID-19.

The associations between AKI, proteinuria, and hematuria in hospitalized patients before COVID-19 is well-documented. For example, in-hospital AKI is associated with proteinuria, and this relationship increases with severity of AKI, possibly leading to CKD [23, 24]. Hematuria was also reported to be associated with AKI, both as a possible cause through tubular obstruction and heme pigment-induced kidney injury as well as a consequence of AKI due to renal inflammation $[25,26]$. Data on the prevalence, risk, and associated outcomes related to proteinuria and hematuria in patients with COVID-19 are more limited. While we excluded all patients with urinary tract infection, the etiology of hematuria and proteinuria remains unclear. Due to precautionary measures taken in the severe COVID-19 outbreak in March and April 2020, most patients did not have kidney imaging or biopsy done for diagnostic purposes. Nonetheless, our data provide important prognostic information to all healthcare providers in managing patients with COVID-19 during hospitalization. Our data also provide information to researchers to investigate causes of hematuria and proteinuria in patients with COVID-19 and test the impact of potential preventive therapies in future waves of this pandemic.

In our study, we also noted a $27 \%$ prevalence of AKI, which is less than reported by other US cohorts, possibly due to the differences in baseline characteristics of patients. For example, compared to Hirsch et al. [9], our cohort was younger and had a lower proportion of diabetics and hypertensives. History of hypertension was significantly associated with both admission and in-hospital AKI in our cohort, as reported in other studies of AKI in COVID-19 [9]. It is not clear why the history of hypertension increases AKI risk in COVID-19. Baseline vascular disease might be contributing to impaired autoregulation in the kidney, thereby placing hypertensive patients at higher risk of AKI from the vascular and hemodynamic abnormalities noted in COVID-19. While admission AKI was not associated with adverse outcomes in our cohort, patients with in-hospital AKI had a significantly higher risk of death compared to those without in-hospital AKI, consistent with other studies $[8,9,14]$. The importance of AKI in hospitalized patients with COVID-19 is reinforced by our finding that recovery was associated with a major reduction in mortality risk.

SARS-CoV-2 utilizes angiotensin-converting enzyme 2 (ACE2) as a port of entry into cells [27], which is found mainly in the proximal tubules and to a lesser extent in podocytes [28]. Recent pathology data from autopsy and kidney biopsy studies are against direct viral toxicity being a primary mechanism of kidney injury in COVID-19 [29-31]. These studies suggest acute tubular injury $[29,31]$ and cytokine-mediated hyperimmune response [30] as potential

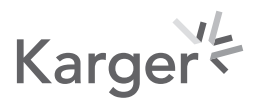


mechanisms of kidney injury. It has been postulated that SARS-CoV-2 binding to ACE2 on endothelial cells induces an inflammatory response leading to a systemic vasculitis-like syndrome [32]. Other postulated hypotheses for kidney injury include angiotensin 2 pathway activation, dysregulation of complement, hypercoagulation, and microangiopathy [33]. Severe inflammation of the kidney $[34,35]$ could contribute to the hematuria and proteinuria. In addition, traditional risk factors such as sepsis, hypotension, and nephrotoxic drugs might contribute to the acute tubular injury.

This study also has significant limitations. It was a single-center study with a small cohort size. We had incomplete data for inflammation as well as for proteinuria and hematuria, thereby reducing the cohort sizes in those analyses. We also were unable to distinguish patients who had preexisting proteinuria and hematuria prior to presentation (preexisting CKD) from those who had it new-onset on admission (acute kidney impairment as a part of COVID-19) due to lack of previous urinalysis in the EHR in most patients. We did not have data on the severity of hematuria or proteinuria either. We could not discern the etiologies of proteinuria and hematuria in this study. The majority of the patients in our cohort were Caucasians, limiting generalizations to other racial groups. Finally, we did not have urine output data available for accurate analysis, and the definition of AKI was limited to $\mathrm{SCr}$ measures only.

The biggest strength of our study is that all patients had a 100\% disposition (death or discharged alive) to avoid the pitfall in previously published studies with a large number of patients who remained "active" in the hospital. Another key strength is that we conducted a separate analysis of admission and in-hospital kidney abnormalities, unlike previous studies that did not tease out this difference. Other strengths include adjusting for multiple covariates and investigating multiple markers of inflammation.

In conclusion, hematuria and proteinuria, both at the time of admission and new-onset during hospitalization, are associated with adverse outcomes in hospitalized patients with COVID-19. Further studies are required to investigate the etiologies of these kidney abnormalities.

\section{Statement of Ethics}

The research was conducted in compliance with the guidelines for human studies in accordance with the World Medical Association Declaration of Helsinki. Informed consent was waived since this was a retrospective study without patient identifiers. The study protocol was approved by the Stony Brook University Institutional Review Board.

\section{Conflict of Interest Statement}

The authors have no conflicts of interest to declare.

\section{Funding Sources}

None. 


\section{Author Contributions}

I. Chaudhri, S.K. Mallipattu, and F.M. Koraishy designed the study. I. Chaudhri, O. Bolotova, J. Yoo, and S. Dhaliwal carried out the data extraction. I. Chaudhri, E. Taub, H. Sahib, R. Moffitt, M. Hoai, R.R. Annadi, S.K. Mallipattu, and F.M. Koraishy analyzed the data. I. Chaudhri, E. Taub, H. Sahib, S.K. Mallipattu, and F.M. Koraishy made the tables. I. Chaudhri, S.K. Mallipattu, and F.M. Koraishy drafted the paper. All authors revised the paper and approved the final version of the manuscript.

\section{References}

1 WHO. Coronavirus disease (COVID-19) pandemic. 2020. Available from: www.who.int/emergencies/ diseases/novel-coronavirus-2019 [cited 06/24/2020].

2 Li X, Ma X. Acute respiratory failure in COVID-19: is it "typical” ARDS? Crit Care. 2020 May;24(1):198.

3 Li JW, Han TW, Woodward M, Anderson CS, Zhou H, Chen YD, et al. The impact of 2019 novel coronavirus on heart injury: A Systematic review and Meta-analysis. Prog Cardiovasc Dis. 2020 Jul-Aug;63(4):518-24.

4 Wilson MP, Jack AS. Coronavirus disease 2019 (COVID-19) in neurology and neurosurgery: A scoping review of the early literature. Clin Neurol Neurosurg. 2020 Jun;193:105866.

5 Fogarty H, Townsend L, Ni Cheallaigh C, Bergin C, Martin-Loeches I, Browne P, et al. COVID19 coagulopathy in Caucasian patients. Br J Haematol. 2020 Jun;189(6):1044-9.

6 Bouaziz JD, Duong T, Jachiet M, Velter C, Lestang P, Cassius C, et al. Vascular skin symptoms in COVID-19: a French observational study. J Eur Acad Dermatol Venereol. 2020 doi: 10.1111/jdv.16544 [Epub ahead of print].

7 McGonagle D, Sharif K, O’Regan A, Bridgewood C. The Role of Cytokines including Interleukin-6 in COVID-19 induced Pneumonia and Macrophage Activation Syndrome-Like Disease. Autoimmun Rev. 2020 Jun;19(6): 102537.

8 Cheng Y, Luo R, Wang K, Zhang M, Wang Z, Dong L, et al. Kidney disease is associated with in-hospital death of patients with COVID-19. Kidney Int. 2020 May;97(5):829-38.

9 Hirsch JS, Ng JH, Ross DW, Sharma P, Shah HH, Barnett RL, et al.; Northwell COVID-19 Research Consortium; Northwell Nephrology COVID-19 Research Consortium. Acute kidney injury in patients hospitalized with COVID-19. Kidney Int. 2020 Jul;98(1):209-18.

10 Richardson S, Hirsch JS, Narasimhan M, Crawford JM, McGinn T, Davidson KW, et al.; the Northwell COVID-19 Research Consortium. Presenting Characteristics, Comorbidities, and Outcomes Among 5700 Patients Hospitalized With COVID-19 in the New York City Area. JAMA. 2020 May;323(20):2052-9.

11 Wang L, Li X, Chen H, Yan S, Li D, Li Y, et al. Coronavirus disease 19 infection does not result in acute kidney injury: an analysis of 116 hospitalized patients from Wuhan, China. Am J Nephrol. 2020;51(5):343-8.

12 Chen T, Wu D, Chen H, Yan W, Yang D, Chen G, et al. Clinical characteristics of 113 deceased patients with coronavirus disease 2019: retrospective study. BMJ. 2020 Mar;368:m1091.

13 Arentz M, Yim E, Klaff L, Lokhandwala S, Riedo FX, Chong M, et al. Characteristics and Outcomes of 21 Critically Ill Patients With COVID-19 in Washington State. JAMA. 2020 Apr;323(16):1612-4.

14 Pei G, Zhang Z, Peng J, Liu L, Zhang C, Yu C, et al. Renal Involvement and Early Prognosis in Patients with COVID-19 Pneumonia. J Am Soc Nephrol. 2020 Jun;31(6):1157-65.

15 Liu R, Ma Q, Han H, Su H, Liu F, Wu K, et al. The value of urine biochemical parameters in the prediction of the severity of coronavirus disease 2019. Clin Chem Lab Med. 2020 Jun;58(7):1121-4.

16 Acute Kidney Injury Work Group. KDIGO Clinical Practice Guideline for Acute Kidney Injury. Kidney Int Suppl. 2012;2(1):1-138.

17 Mehta RL, Kellum JA, Shah SV, Molitoris BA, Ronco C, Warnock DG, et al.; Acute Kidney Injury Network. Acute Kidney Injury Network: report of an initiative to improve outcomes in acute kidney injury. Crit Care. 2007; 11(2):R31.

18 Chawla LS, Bellomo R, Bihorac A, Goldstein SL, Siew ED, Bagshaw SM, et al.; Acute Disease Quality Initiative Workgroup 16. Acute kidney disease and renal recovery: consensus report of the Acute Disease Quality Initiative (ADQI) 16 Workgroup. Nat Rev Nephrol. 2017 Apr;13(4):241-57.

19 Levey AS, Stevens LA, Schmid CH, Zhang YL, Castro AF 3rd, Feldman HI, et al.; CKD-EPI (Chronic Kidney Disease Epidemiology Collaboration). A new equation to estimate glomerular filtration rate. Ann Intern Med. 2009 May;150(9):604-12.

20 Jenny NS, Brown ER, Detrano R, Folsom AR, Saad MF, Shea S, et al. Associations of inflammatory markers with coronary artery calcification: results from the Multi-Ethnic Study of Atherosclerosis. Atherosclerosis. 2010 Mar;209(1):226-9.

21 Chaudhri I, Koraishy FM, Bolotova O, Yoo J, Marcos LA, Taub E, et al. Outcomes associated with the use of reninangiotensin-aldosterone system blockade in hospitalized patients with SARS-CoV-2 infection. Kidney360. 2020 Aug:1(8);801-9. 
22 R Core Team. R: A language and environment for statistical computing. Vienna, Austria: R Foundation for Statistical Computing; 2019.

23 Hsu CY, Hsu RK, Liu KD, Yang J, Anderson A, Chen J, et al.; Chronic Renal Insufficiency Cohort (CRIC) Study Investigators and the Assessment, Serial Evaluation, and Subsequent Sequelae of Acute Kidney Injury (ASSESSAKI) Study. Impact of AKI on Urinary Protein Excretion: Analysis of Two Prospective Cohorts. J Am Soc Nephrol. 2019 Jul;30(7):1271-81.

24 Parr SK, Matheny ME, Abdel-Kader K, Greevy RA Jr, Bian A, Fly J, et al. Acute kidney injury is a risk factor for subsequent proteinuria. Kidney Int. 2018 Feb;93(2):460-9.

25 Moreno JA, Martín-Cleary C, Gutiérrez E, Toldos O, Blanco-Colio LM, Praga M, et al. AKI associated with macroscopic glomerular hematuria: clinical and pathophysiologic consequences. Clin J Am Soc Nephrol. 2012 Jan; $7(1): 175-84$.

26 Moreno JA, Sevillano Á, Gutiérrez E, Guerrero-Hue M, Vázquez-Carballo C, Yuste C, et al. Glomerular Hematuria: Cause or Consequence of Renal Inflammation? Int J Mol Sci. 2019 May;20(9):E2205.

27 Astuti I, Ysrafil. Severe Acute Respiratory Syndrome Coronavirus 2 (SARS-CoV-2): an overview of viral structure and host response. Diabetes Metab Syndr. 2020 Jul-Aug;14(4):407-12.

28 Ye M, Wysocki J, William J, Soler MJ, Cokic I, Batlle D. Glomerular localization and expression of Angiotensinconverting enzyme 2 and Angiotensin-converting enzyme: implications for albuminuria in diabetes. J Am Soc Nephrol. 2006 Nov;17(11):3067-75.

29 Golmai P, Larsen CP, DeVita MV, Wahl SJ, Weins A, Rennke HG, et al. Histopathologic and Ultrastructural Findings in Postmortem Kidney Biopsy Material in 12 Patients with AKI and COVID-19. J Am Soc Nephrol. 2020 Sep;31(9):1944-7.

30 Kudose S, Batal I, Santoriello D, Xu K, Barasch J, Peleg Y, et al. Kidney Biopsy Findings in Patients with COVID-19. J Am Soc Nephrol. 2020 Sep;31(9):1959-68.

31 Santoriello D, Khairallah P, Bomback AS, Xu K, Kudose S, Batal I, et al. Postmortem Kidney Pathology Findings in Patients with COVID-19. J Am Soc Nephrol. 2020 Sep;31(9):2158-67.

32 Sardu C, Gambardella J, Morelli MB, Wang X, Marfella R, Santulli G. Hypertension, Thrombosis, Kidney Failure, and Diabetes: Is COVID-19 an Endothelial Disease? A Comprehensive Evaluation of Clinical and Basic Evidence. J Clin Med. 2020 May; 9(5):E1417.

33 Batlle D, Soler MJ, Sparks MA, Hiremath S, South AM, Welling PA, et al.; COVID-19 and ACE2 in Cardiovascular, Lung, and Kidney Working Group. Acute Kidney Injury in COVID-19: Emerging Evidence of a Distinct Pathophysiology. J Am Soc Nephrol. 2020 Jul;31(7):1380-3.

34 Wang D, Hu B, Hu C, Zhu F, Liu X, Zhang J, et al. Clinical characteristics of 138 hospitalized patients with 2019 novel coronavirus-infected pneumonia in Wuhan, China. JAMA. 2020 Mar;323(11):1061-9.

35 Perico L, Benigni A, Remuzzi G. Should COVID-19 Concern Nephrologists? Why and to What Extent? The Emerging Impasse of Angiotensin Blockade. Nephron. 2020;144(5):213-21. 